\title{
Decompressive Craniectomy for Traumatic Brain Injury
}

Can. J. Neurol. Sci. 2011; 38: 541-542

Decompressive craniectomy, the removal of a large bone flap to relieve brain swelling has a long and chequered history. In 1901 Kocher described 'If there is no cerebrospinal fluid (CSF) pressure, but brain pressure exists, then pressure relief must be achieved by opening the skull'. The operation has been applied to several diverse pathologies including traumatic brain injury, subarachnoid haemorrhage, intra-cerebral haemorrhage, encephalitis and rarer causes of brain swelling including Acute Disseminated Encephalomyelitis. Decompressive craniectomy following traumatic brain injury was very popular in the 1970 s but subsequently enthusiasm waned due to concerns over clinical outcome. There was a renaissance in the 1990s and since then there have an increasing number of studies presenting the results of this operation in various observational series of patients.

In this edition of The Canadian Journal of Neurological Sciences (CJNS), Al-Jishi et $\mathrm{al}^{1}$ present the results of decompressive craniectomy in 70 head injured patients over a four year period. What is different about this study? The value of this paper is that it clearly distinguishes between outcome following primary and secondary decompressive craniectomy. Primary decompressive craniectomy is craniectomy performed emergently (within hours of trauma) usually in conjunction with removal of a mass lesion. Secondary decompressive craniectomy is usually performed in the ensuing days following trauma in order to control brain swelling and raised intracranial pressure not responding to medical treatment. The results showed a significant difference in the mechanism of injury, the pupil abnormalities and Marshall computed tomogram (CT) grade between primary and secondary decompressive craniectomy. There was a markedly significant difference in outcome with primary decompressive craniectomy showing $45.5 \%$ good outcome and $40.9 \%$ mortality versus secondary decompressive craniectomy with $73.1 \%$ good outcome and $15.4 \%$ mortality. These latter figures for secondary decompressive craniectomy are exceptionally good when compared with the literature and are a testament to good patient selection, surgery and intensive care both pre and post-operatively. The authors applied sensible criteria for both primary and secondary decompressive craniectomy.

However, concerns remain as with other series that the operation is being performed unnecessarily in patients deemed to have a good prognosis with medical treatment alone and that the operation may save life but at the expense of producing vegetative state and severe disability. In this series this latter concern is less than that of other series with a severe disability / vegetative state rate of $13.6 \%$ for the primary group and $11.5 \%$ for the secondary group. However, as the authors acknowledge even patients with a "good outcome" may have ongoing problems which are not detected by the Glasgow Outcome Score.
In order to explore and refine the indications for decompressive craniectomy there are two multicentre randomised studies of decompressive craniectomy following traumatic brain injury: RESCUEicp ${ }^{2}$ and DECRA ${ }^{3}$.

Decompressive Craniectomy in Diffuse Traumatic Brain Injury (DECRA) run from Melbourne, Australia aimed to test the hypothesies that early (within 72 hours of injury) bifrontotemporoparietal decompressive craniectomy in adults under the age of 60 years results in an improvement in outcome compared to ongoing medical treatment alone. The study has an intracranial pressure (ICP) threshold of $20 \mathrm{mmHg}$ and applies to patients with diffuse injury only. The study recruited 155 patients and the results have just been published, interestingly showing that early decompressive craniectomy reduced ICP and length of stay but patients undergoing the operation had worse scores on the Extended Glasgow Outcome Scale and similar mortality to those not undergoing decompression.

Randomised evaluation of Surgery with Craniectomy for uncontrollable elevation of ICP (RESCUEicp) (www.RESCUE icp.com) run from Cambridge, United Kingdom (UK) aims to address the hypothesis that the application of decompressive craniectomy to head-injured patients with raised and refractory ICP results in improvement in outcome compared to optimal medical management. The study is on-going, has recruited 300 / 400 patients as of April 2010 with an ICP threshold of $25 \mathrm{mmHg}$, contusions accepted and no time limit post injury but time controlled in terms of duration of ICP not controlled by medical treatment.

We strongly believe that the jury remains out on the indications and timing of this operation, particularly following the results of the DECRA. Further investigations, including randomised studies (completion of RESCUEicp) are required in order to provide a definitive answer and avoid the roller coaster ride of enthusiasm / nihilism that this operation has encountered over the past 40 years. If patients are not randomised it is at least essential that the results are closely monitored as undertaken by this study. However, this and the other contemporary series in the literature would benefit from improvements in terms of the assessment of outcome, with in addition to Glasgow Outcome Score, the assessment of other measures such as quality of life and neuropsychological measures. This will continue to enhance our knowledge of this common and often disabling condition.

Peter Hutchinson Reader in Neurosurgery University of Cambridge United Kingdom 


\section{REFERENCES}

1. Al-Jishi A, Saluja RS, Al-Jehani H, et al. Primary or secondary decompressive craniectomy: different indication and outcome. Can J Neurol Sci. 2011; 38(4):612-20.

2. Hutchinson PJ, Corteen E, Czosnyka M, et al. Decompressive craniectomy in traumatic brain injury: the randomised multicenter RESCUEicp study (www.RESCUEicp.com). Acta Neurochir Suppl. 2006; 96: 17-20.

3. Cooper DJ, Rosenfeld JV, Murray L, et al. The DECRA Trial Investigators and the Australian and New Zealand Intensive Care Society Clinical Trials Group. Decompressive craniectomy in diffuse traumatic brain injury. N Engl J Med. 2011 Mar 25. [Epub a head of print]. 